\title{
JUAN ESTRUCH
}

\section{LA INNOVACION RELIGIOSA}

Acaso el problema fundamental que plantea toda empresa sociológica sea el de la relativización de los hechos que estudia. El mundo de las evidencias colectivas, de lo «dado por supuesto», del "ça-va-de-soi», del «taken-for-granted», de aquello que no cabe poner en tela de juicio, se convierte - desde una perspectiva sociológica - en un mundo socialmente construido y explicable. La diferencia entre una sociología espontánea y una auténtica sociología estriba precisamente en el hecho de que la segunda no exime del proceso de análisis a aquellas realidades que la primera sigue dando por supuestas y legitima con el recurso a una «etiqueta» científica ${ }^{1}$. De ahí las reticencias y suspicacias que en ciertos ambientes siguen rodeando a toda empresa sociológica. En efecto, la relativización a la que la sociología somete a toda realidad lógicamente insecuriza a quien la había dado por supuesta y la había considerado como incuestionable.

En el caso de la sociología de la religión, e independientemente del hecho de que el sociólogo afirme o niegue en su fuero interno la existencia de aspectos «meta-sociales» en la religión, ello supone que su empresa necesariamente ha de conducirle a la relativización del campo específico de la realidad social que se ha propuesto investigar, y que resulta ser uno de los más difícilmente relativizables: la religión. Sin embargo, y hechas todas las salvedades que se imponen en el sentido de recordar y reafirmar

1. Cf. P. Bourdiev, J.-Cl. Chamboredon, J.-Cu. Passeron, le métier de sociologue, París, Mouton-Bordas, 1968, págs. 37-47. 
la parcialidad intrínseca de todo "punto de vista» - y por consiguiente también del punto de vista sociológico-, su enfoque no puede ser más que el del estudio de la religión en tanto que fenómeno social, proyección humana, realidad socialmente construida.

A partir de una perspectiva vinculada a una sociología del conocimiento o una sociología de la cultura, este artículo se propone el establecimiento de un marco teórico de referencia para un estudio sociológico del fenómeno religioso. Preside, en efecto, el trabajo una voluntad de hacer una contribución a uno de los campos que mayormente parecen haber quedado relegados a un segundo plano, particularmente en España: la sociología de la religión. Relegación a un segundo plano que resulta tanto más injustificable cuanto que lo religioso sigue siendo un factor de capital importancia para la comprensión sociológica de una realidad como la española.

Esta aparente contradicción parece, sin embargo, explicable a partir de un análisis del proceso de desarrollo de la sociología de la religión como disciplina. Efectivamente, a una primera etapa caracterizada por el estudio sociológico de la religión en su calidad de fenómeno de importancia para la comprensión de una sociedad determinada -es decir, estudio de lo religioso por motivaciones sociológicas-, sucede en Europa una segunda fase que esquemáticamente cabría caracterizar más bien como de recurso a categorías sociológicas por motivaciones de tipo eminentemente religioso ${ }^{2}$. La proliferación de encuestas sobre los comportamientos religiosos, de investigaciones al servicio del «management» de la institución eclesiástica, de estudios sociográficos sobre la coyuntura religiosa, dentro siempre de una perspectiva claramente eclesiocéntrica, ha conducido lógicamente a un empobrecimiento de la teoría sociológica de la religión $\mathrm{y}$, en último término, a su marginación con respecto a las demás ramas de la sociología. El presente trabajo pretende contribuir a la superación de esta etapa de sociología «eclesiástica» o «sociología religiosa», para entroncar con la primitiva tradición y fomentar un nuevo desarrollo de una auténtica «sociología de la religión».

Esta clara opción en pro de una sociología de la religión (por contraposición a la sociología religiosa de la segunda fase del proceso), combinada con el hecho de la carencia casi absoluta de material teórico sobre los fenómenos de cambio aplicados a lo religioso, obliga a hacer del tema de la innovación religiosa un tratamiento eminentemente teórico, susceptible de proporcionar un marco de referencia a partir del cual quepa elaborar

2. Véase al respecto el artículo de P. Berger y T. Luckmann, «Sociology of Religion and Sociology of Knowledge», Sociology and Social Research, Vol. 47, $\mathbf{n} .{ }^{\circ}$, 1963, págs. 417-427. 
una serie de hipótesis a verificar (o a invalidar) en investigaciones empíricas ulteriores.

El análisis, circunscrito en el espacio a la sociedad occidental, y en el tiempo al momento presente, avanza paralelamente en dos frentes complementarios: elaboración del marco teórico de referencia por una parte, y análisis de la literatura sociológica sobre el tema y sobre problemas conexos, por otra. En tres etapas sucesivas pasaremos de la teoría sociológica $(\S 1)$ a la sociología de la religión ( $\$ 2$ ), y de ésta a una posible aplicación del modelo teórico a ciertos aspectos de la Iglesia como institución (§3). En efecto, si la perspectiva sociológica adoptada implica que se rechace de entrada toda forma de «eclesiocentrismo» en el enfoque del trabajo, la suprema especialización institucional de la religión en forma de Iglesia dentro de la sociedad occidental ${ }^{3}$ obliga a buscar dentro de un marco eclesiástico las ilustraciones a las que recurre la elaboración teórica, así como las aplicaciones que de ella quepa hacer.

\section{Delimitación Del Tema Y elaboración DEL MARCo TEÓRICO DE REFERENCIA}

La palabra innovación es definida en términos muy semejantes por los distintos diccionarios y enciclopedias consultados, en idiomas diferentes: todas las definiciones concuerdan en (y generalmente se limitan a) afirmar que se trata de la introducción de una novedad en algo ya existente o establecido. Por otra parte, todas ellas son perfectamente neutras, de tal modo que a ese nivel de definiciones "oficiales» el término innovación no aparece dotado de connotaciones afectivas de signo positivo o negativo.

En el empleo corriente de la palabra en el lenguaje habitual, por el contrario, el término no es ya tan neutro. Dentro de un contexto religioso ( $y$ en particular en la Iglesia católica), innovación podría ser una de las palabras clave en torno a la cual se polarizan las tendencias contradictorias actuales (junto con otros términos próximos, tales como renovación, aggiornamento, etc.). Para los grupos que luchan por la innovación como para quienes luchan contra ella, la carga emotiva del término es manifiesta. Huelga decir que nosotros debemos emplear aquí el concepto sin darle connotación afectiva alguna, y sin tomar partido por ninguno de los grupos para quienes la palabra es una especie de bandera - a izar o a arriar, según los casos.

3. Cf. T. Luckmann, «On Religion in Modern Society: Individual Consciousness, World View, Institution», Journal for the Scientific Study of Religion, vol. 2, n. ${ }^{\circ}$, 1963, pág. 158. 
De todos modos, en su empleo más habitual la palabra innovación no suele ir asociada a la esfera de lo religioso. Habiendo procedido a un experimento de asociación de palabras entre diez personas (universitarios), que espontáneamente debían asociar el término innovación a otros, en nueve casos apareció (casi siempre entre las tres primeras) la palabra progreso, y en siete la palabra técnica (o tecnología). En tercer lugar venía el término ordenador (o computador, cerebro electrónico, etc.). Dentro de una lógica diversidad de las respuestas puede observarse claramente la aparición de una sorprendente coherencia en este tipo de asociaciones. La innovación tiende a asociarse, en una percepción común y espontánea, con el progreso, y fundamentalmente con el progreso de tipo tecnológico. Ello es tanto más interesante cuanto que, como en seguida veremos, esta percepción se aproxima bastante al punto de vista de la literatura sociológica existente sobre la innovación. Lo cual plantea necesariamente de nuevo el problema de las connotaciones afectivas ya que, efectivamente, de modo implícito la innovación asociada a la noción de progreso lleva una connotación de signo positivo. Por otra parte, esa percepción espontánea de la innovación rara vez hace intervenir elementos de tipo religioso en el contenido del concepto. $\mathrm{El}$ análisis de la literatura sociológica sobre el tema habrá de permitir preguntarnos por qué. Puesto que, como se comprenderá, sí emprendemos un estudio sobre la innovación religiosa es porque partimos de la hipótesis de que el tema nos va a permitir estudiar otros problemas que el de la adquisición, por ejemplo, de un ordenador por el Vaticano con el fin de racionalizar el cálculo de las nóminas de todos los funcionarios y empleados de la Santa Sede.

El análisis de la literatura sociológica existente sobre la innovación permite darse cuenta de que el concepto se asocia básicamente a productos u objetos nuevos por una parte, o a nuevas técnicas o conocimientos empíricos por otra, con una eventual distinción entre la innovación y su difusión. Paralelamente, los campos concretos de la sociología a los que se aplica esta literatura son sobre todo dos: la sociología rural y la sociología industrial. En el primer caso, las investigaciones parten de una preocupación por los problemas de la innovación en relación con la problemática de la racionalización de las técnicas agrícolas y de la adopción de este tipo de novedades (difusión); en el segundo caso, la innovación va estrechamente asociada a nociones como la tecnología, el «management» de empresas y organizaciones, y la personalidad del líder o manager en tanto que innovador. El análisis crítico de esta literatura sobre la innovación exige un estudio de los presupuestos implícitos de todas estas investigaciones, y debería tener en cuenta los siguientes elementos: 
a) Innovación y progreso. - La percepción no sociológica de la innovación tiende a asociar espontáneamente, según vimos, esta noción a la de progreso, y en especial el progreso de tipo tecnológico. Ello parece coincidir en buena medida con la definición (implícita o explícita) que de la innovación suelen dar los sociólogos y los antropólogos. En efecto, tanto si se trata del descubrimiento de un objeto nuevo, o de un invento, como de la adopción de una nueva técnica, la noción de progreso se halla contenida de modo subyacente en la forma misma de enfocar la cuestión. Pero, chasta qué punto puede generalizarse semejante noción, extendiéndola a otros campos? ¿Hasta qué punto, por ejemplo, es aplicable en el caso de la religión?

b) Innovación y difusión. - En segundo lugar, la problemática de la difusión parece comportar asimismo ciertos presupuestos implícitos. La innovación es percibida como algo positivo, que se trata de hacer aceptar a los demás. Esta sociología de la difusión se convierte así de algún modo en una forma de «management», de instrumento al servicio del que dispone del poder (del «decision-maker», para utilizar la terminología propia de este sector). Tanto en el mundo rural como en el industrial o empresarial se supone que el innovador se halla en situación de poder y ha de vencer la resistencia, ya de una masa apegada a sus tradiciones, ya de una rutina burocrática que se opone igualmente a la adopción de novedades. También en este caso cabe preguntarse hasta qué punto se aplica este esquema a todos los campos. En la esfera religiosa, por ejemplo, ¿no cabría suponer un tipo de innovación promovida por ciertos grupos que no se hallan en situación de poder? ¿O una innovación que respondiera a unas ciertas necesidades sentidas por la masa, y que se enfrentara con la resistencia del poder? ¿En qué medida la situación actual de la institución religiosa responde más a este segundo modelo que al del líder-manager-innovador?

c) Innovación y sistema de conocimentos empíricos. - La explicación del dilema radica probablemente en el hecho de que toda la literatura existente sobre la innovación se sitúa al nivel de los conocimientos empíricos (descubrimientos, inventos, introducción de técnicas nuevas) o de los medios. propuestos para alcanzar unos objetivos determinados (difusión de progresos tecnológicos, management industrial y empresarial). Nos hallamos esencialmente a nivel de lo racional. Ahora bien, si bien es cierto que ese nivel adquiere en la sociedad contemporánea una importancia cada vez mayor, no por ello es el único nivel posible en el que quepa situarse. Al lado del nivel de lo racional está el nivel de lo razonable; al lado de lo que llama- 
remos sistema de conocimientos empíricos está lo que denominaremos sistema de legitimación. $Y$ si en el sistema de conocimientos empíricos imperan los criterios de racionalidad y eficacia, los criterios determinantes del sistema de legitimación no son los mismos. Nuestra hipótesis es que en el caso de la religión el sistema de legitimación desempeña un papel de importancia fundamental y que, por consiguiente, una teoría de la innovación basada únicamente en elementos pertenecientes al sistema de conocimientos empíricos es de todo punto insuficiente en el marco de una sociología de la religión.

Un esquema teórico tradicional, cual el de una tipología bipolar basada en el criterio del proceso de diferenciación de la sociedad, permite analizar las distintas modalidades de inserción social y de institucionalización de la religión en el paso de una sociedad no diferenciada a una sociedad diferenciada. Tomando como variables el predominio de la segmentación o de la especialización, y el predominio del control ecológico o de un control de tipo no ecológico ${ }^{4}$, la sociedad no diferenciada se definiría como aquella que se compone de un conjunto de pequeños grupos en los que el individuo se halla constantemente inserto, de suerte que todas sus actividades se ven sometidas a una observabilidad directa (control ecológico); por otra parte, este tipo de sociedad tiende a organizarse como una pirámide estable y jerarquizada, a partir de pequeñas unidades concéntricas y polivalentes integradas las unas a las otras (segmentación, es decir, repetición de un mismo modelo cultural en distintas unidades similares). Frente a este modelo, una sociedad diferenciada se caracterizaría más bien por una disociación entre la vida f́amiliar y recreativa por una parte, y la vida profesional por otra; existe normalmente incluso una distancia espacial entre la primera, convertida en centro de consumo, y la segunda, centro de producción: el control ecológico se modifica y se reduce con respecto al primer modelo. Por otra parte, la segmentación es en buena parte substituida por la especialización, entendida como el desarrollo de grandes organizaciones con objetivos especializados, que impiden la repetición de un mismo modelo cultural en una multiplicidad de unidades para dar lugar a una sola unidad de grandes dimensiones y caracterizada por la división del trabajo.

En el paso de una sociedad no diferenciada a una sociedad diferenciada, la religión deja de ser un elemento decisivo para la explicación de la organización y el desarrollo del sistema cultural. La mayor autonomía de

4. Cf. J. Remy, «La religion dans une société pluraliste», Social Compass, Vol. $13, n$. 5-6, especialmente págs. 381-385. 
cada uno de los elementos de ese sistema hace que la religión juegue un papel cada vez más residuario a nivel de los conocimientos empíricos y las normas. En otros momentos históricos la institución religiosa había podido desempeñar a ese nivel un rol que actualmente aparece como un rol de substitución: la religión ofrecía entonces, a la vez que un sistema de creencias y una serie de valores u objetivos a perseguir, un conjunto de normas o medios relativamente indisociables de ellos. En una sociedad diferenciada, por el contrario, el nivel de los conocimientos empíricos y de las normas puede desarrollarse con independencia cada vez mayor con respecto a la religión.

Nos hallamos, pues, ante la combinación de dos factores relacionados entre sí y que nos parecen situar básicamente la posición de la religión en una sociedad diferenciada:

1) La autonomía creciente de cada uno de los elementos del sistema cultural, y la independización del sistema de conocimientos empíricos con respecto a la religión, ponen de manifiesto cuál es la especificidad de la institución religiosa: a saber. su carácter de instancia legitimadora de unos valores, y no ya una intervención decisiva a todos los niveles del sistema cultural.

2) Por otra parte, incluso a este nivel de las legitimaciones y los valores, y del vínculo que la religión establece de modo específico entre unas y otros, la institución religiosa ha perdido el monopolio de que antes gozaba, por el hecho de la coexistencia de distintas legitimaciones religiosas y, más aun, por la coexistencia de un sistema de legitimación religiosa con un sistema de legitimación que excluye la religión.

Ello determina la orientación a dar a la elaboración de una teoría sociológica de la innovación religiosa: por una parte, en efecto, habrá que estudiar cómo se presenta la innovación según afecte a las legitimaciones, a los valores o a las normas. Siendo la zona de las legitimaciones y los valores la que más precisamente caracteriza a la institución religiosa, las teorías sociológicas de la innovación anteriormente consideradas ( $\mathrm{y}$ aplicables a áreas tales como la sociología rural y la sociología industrial) se revelan necesariamente insuficientes para nuestra perspectiva, en la medida en que fundamentalmente se basan en elementos pertenecientes al sistema de conocimientos empíricos. En segundo lugar, habrá que preguntarse hasta qué punto una situación de pluralismo o de pérdida del monopolio que la religión había ostentado a nivel de las legitimaciones puede modificar las condiciones de aparición y de desarrollo de la innovación religiosa. Un examen de la escasa literatura sociológica existente sobre la innovación 
La innovación religiosa

religiosa nos permitirá situar el problema teórico, como paso previo a un intento de análisis de las consecuencias de la inserción de la religión en una sociedad diferenciada sobre la problemática de la innovación, dentro siempre de la perspectiva cultural aquí adoptada.

\section{LA INNOVACIÓN RELIGIOSA}

Si la introducción de una innovación en una sociedad no diferenciada fácilmente puede desequilibrar todo el sistema, dadas las estrechas relaciones de interdependencia existentes entre legitimaciones, valores y normas, dentro de una sociedad diferenciada, en cambio, la mayor autonomía de cada uno de los elementos del sistema cultural hace que una innovación situada a uno de los niveles tenga un impacto mucho menor sobre los otros dos.

Una diferencia fundamental distingue, en efecto, a la innovación situada a nivel de los conocimientos empíricos y las normas, de la innovación que afecta a las legitimaciones y los valores. La distinción antes establecida entre los niveles de lo racional y lo razonable cobra aquí toda su importancia: las normas, al no implicar una adhesión afectiva, son mucho más susceptibles de ser alteradas en función de las circunstancias: pueden ser puestas en tela de juicio a corto plazo, es decir, pueden tolerar una innovación constante. A este nivel del sistema cultural cabría hablar incluso de la posibilidad de una institucionalización de la innovación ${ }^{5}$.

Valores y legitimaciones constituyen, por el contratio, un conjunto de evidencias colectivas, y la implicación afectiva que exigen del grupo que adhiere a ellos hace mucho más difícil la introducción de innovaciones. Una innovación a nivel de las legitimaciones supone una modificación de la justificación que el grupo da de su razón de ser. En cuanto a los valores, el problema es más delicado aún por cuanto se trata de los objetivos mismos que el grupo se fija, y que normalmente no pueden ser puestos en tela de juicio a corto plazo sin que el grupo corra el riesgo de caer en una situación de desequilibrio grave y de crisis de identidad.

Por otra parte, en la medida en que la sociedad diferenciada se caracteriza por un acuerdo global mínimo sobre un cierto número de valores, acuerdo en el que participan los distintos sistemas de legitimación coexistentes en una sociedad determinada, la innovación a nivel de los valores no sólo comporta una dificultad mayor aún si cabe, sino que dependerá

5. Cf. J. RemY, «Innovations et développement des structures», in F. HoutarT y J. RemY, Sacerdoce, autorité et innovation dans l'Eglise, Tours, Mame, 1970, página 235. 
básicamente de la capacidad que cada grupo tenga de imponer unos valores nuevos. Queda con ello planteado un problema realmente fundamental desde nuestra perspectiva de sociología de la religión: chasta qué punto tiene hoy la institución religiosa, dentro de una sociedad diferenciada, la capacidad de proponer unos valores nuevos susceptibles de llegar a imponerse dentro de la sociedad global? thasta qué punto es capaz de proponer unos valores nuevos que puedan llegar a formar parte de este núcleo en torno al cual se constituye un acuerdo general de los distintos sistemas de legitimación? Plantear la cuestión de la motricidad de lo religioso en nuestra sociedad contemporánea equivale de algún modo, efectivamente, a preguntarse por su capacidad de actuar como variable independiente.

Por su parte, un análisis crítico de la literatura sociológica sobre la innovación religiosa nos conduce a plantear prácticamente la misma pregunta. En efecto, el estudio de la teoría weberiana del liderazgo carismático, con sus derivaciones de la rutinización del carisma (Weber ${ }^{6}$, $\mathrm{O}^{\prime} \mathrm{Dea}^{7}$ ) y de las relaciones entre carisma e innovación (Weber ${ }^{8}$, Berger ${ }^{9}$ ); la secuencia de cambio propuesta por Wallace ${ }^{10}$, y la aplicación al fenómeno del cambio religioso de la secuencia de Smelser ${ }^{11}$; la visión parsoniana de la religión como fuente de innovación creadora ${ }^{12}$, la perspectiva neo-evolucionista de Bellah ${ }^{13}$, y la tesis de la adaptación de la religión (en sus vertientes organizacional, ética, y de las formas de las creencias) a las estructuras de la sociedad contemporánea, defendida por Schelsky ${ }^{14}$, inves-

6. Cf. H. Gerth y C. .W Mills, From Max Weber: Essays in Sociology, New York, Oxford University Press, 1958, págs. 245-252 (versión castellana, Ensayos de sociología contemporánea, Barcelona, Martínez Roca, 1972); M. WEBER, Tbe Tbeory of Social and Economic Organization, New York, The Free Press, 1969, pág. 328, págs. 358-363 y págs. 363-373.

7. T. F. O'DEA, «Five Dilemmas in the Institutionalization of Religion», Journal for the Scientific Study of Religion, Vol. 1, n.* 1, 1961, págs. 30-39.

8. Cf. especialmente su obra Le judaïsme antique, París, Plon, 1971.

9. P. L. BERGER, «Chatisma and religious innovation», American Sociological Review, Vol. 28, n. 6, 1963, págs. 940-950.

10. A. WallaCE, «Revitalization Movements», American Antbropologist, Vol. 58, 1956, págs. 264-281.

11. N. J. SMELSER, Social Change in the Industrial Revolution, London, Routledge \& Kegan Paul, 1959, pág. 13.

12. T. PARSONS, "Religion as a Source of Creative Innovation», in J. M. YINGER, Religion, Society and the Individual, New York, Macmillan, 1965, págs. 558-563 (versión castellana, Madrid, Razón y Fe, 1968).

13. En su artículo «Religious Evolution», American Sociological Review, Vol. 29, n. 3, 1964, págs. 358-374.

14. H. SCHELSKY, «Ist die Dauerreflektion institutionalisierbar?», Zeitscbrift für Evangelische Etbik, Vol. 1, 1957, págs. 153-174 (reproducido en versión castellana en el Vol. I de la Sociología de la religión de J. Matr hes, Madrid, Alianza, 1971). 
tigaciones todas ellas que cabe sintetizar en la alternativa de Yinger ${ }^{15}$ : la religión, iniciadora del cambio social, la religión, obstáculo al cambio social, y el cambio religioso como resultado del cambio social, obligan a preguntarse por los presupuestos implícitos de todas estas investigaciones $\mathrm{y}$, concretamente, por la percepción que estos autores tienen de la religión como variable dependiente o como variable independiente.

Se trata en realidad de una doble tradición de enfoque de los fenómenos religiosos -explícita en ciertos casos, implícita las más de las veces- que arranca respectivamente de Marx: el fenómeno religioso como superestructura explicable a partir de la infraestructura socio-económica ${ }^{16}$, y de Weber, que en ciertos casos intenta mantener constante el factor organización económica para tratar la orientación religiosa como variable independiente ${ }^{17}$.

Situados, pues, ante una alternativa que hemos dicotomizado como la del estudio del hecho religioso tratado como variable dependiente o bien como variable independiente, nuestra opción consiste precisamente en no tomar partido por ninguna de las dos tendencias, sino en intentar proceder a un análisis que nos permita determinar en qué medida la religión tiene probabilidades de imponerse como instancia promotora de los esquemas culturales que presiden la orientación de los distintos grupos coexistentes dentro de una sociedad diferenciada. Ello supone dejar abierta la posibilidad de toda una gama de tipos distintos de innovación, cuyos dos extremos teóricos serían la innovación puramente creadora y la innovación meramente adaptativa. Efectivamente, cuanto mayor sea la motricidad del fenómeno religioso dentro del área cultural considerada en su globalidad, tanto mayor será su capacidad de actuar como «fuente de innovación creadora»; por el contrario, la ausencia de semejante motricidad conduciría a considerar a la religión como «sujeta al cambio global». Circunscrito en el espacio y en el tiempo, el análisis no prejuzga en ningún momento, sin embargo, de la capacidad intrínseca de creatividad innovadora de lo religioso, como tampoco de su radical incapacidad y de su necesaria condición adaptativa.

Las variables test elegidas para proceder a semejante análisis son el proceso de secularización y el fenómeno del pluralismo. Definida la secularización como proceso de creciente autonomización de la sociedad con respecto a la dominación de las significaciones y de las instituciones reli-

15. Véanse los capitulos 10 y 11 de la obra de YINGER, Religion, Society and the Individual, ya citada.

16. Cf., por ejemplo, O. KLон R, dir., Religion und Atbeismus beute. Ergebnisse und Aufgaben Marxistischer Religionssoziologie, Berlín, VEB Deutscher Verlag der Wissenschaften, 1966 , págs. 15.16 y págs. 39-40.

17. Cf. la introducción de Parsons a la versión inglesa de la Sociology of Religion de M. Weber, Boston, Beacon Press, 1964, pág. XXII. 
giosas ${ }^{18}$, y el pluralismo como coexistencia de dos o más sistemas de legitimación dentro de una misma sociedad, y considerados ambos como elementos definitorios de la situación del fenómeno religioso dentro de la sociedad occidental contemporánea, su estudio y el análisis de sus consecuencias habrán de permitirnos afirmar en qué medida tiende hoy la religión a actuar como variable dependiente o como variable independiente.

La temática de la secularización ha sido abordada desde perspectivas muy distintas por los diferentes sociólogos que se han ocupado del problema. Sirviéndonos del esfuerzo de sistematización de estos distintos enfoques realizado por Shiner ${ }^{19}$, la secularización puede ser considerada como:

a) ocaso de la religión - pérdida de influencia de la doctrina, las instituciones y los valores religiosos que en otro tiempo habían sido dominantes dentro de la sociedad;

b) conformación de la religión al mundo, o mundanización de la religión;

c) desacralización del mundo - el mundo queda progresivamente despojado de su carácter sacral a medida que el hombre y la naturaleza van convirtiéndose en objeto de explicaciones y manipulaciones de tipo tacionalcausal (visión derivada de la noción weberiana de Entzauberung, o desencantamiento del mundo) ${ }^{20}$;

d) independización de la sociedad frente a lo religioso - la sociedad, desacralizada o liberada de las estructuras sacrales impuestas por la esfera religiosa, se autonomiza;

e) transposición de las creencias y modelos de conducta de la esfera religiosa a la esfera secular -incorporación de ciertos aspectos de la esfera religiosa a un contexto antropocéntrico, tras su desvinculación de un contexto sagrado anterior.

Cada una de estas dimensiones de la secularización se traduciría, dentro del contexto de nuestra problemática y a partir del marco de refetencia previamente elaborado, como sigue:

a) deja de insistirse en los elementos más directamente controlables de la religión;

18. Cf. P. Berger y T. Luckmann, «Aspects sociologiques du pluralisme», Archives de Sociologie des Religions, $\mathrm{n}^{\circ} 23,1967$, pág. 118.

19. L. SHINER, «The Meanings of Seculatization», Internationales Jabrbuch für Religionssoziologie, Vol. 3, 1967, págs. 51-59.

20. Cf. H. Gert h y C. W. Minis, From Max Weber, op. cit., pág. 155. 
La innovación religiosa

b) la religión se ve obligada a legitimar los valores y los esquemas culturales dominantes en la sociedad;

c) la religión pierde el monopolio que anteriormente había ostentado en la definición de las legitimaciones;

d) en vez de desempeñar un papel fundamental en la integración de la sociedad (esfera pública), la institución religiosa se convierte en institución a la que el individuo se puede o no adherir para dar sentido a su actuación (legitimación y esfera privada);

e) otros sistemas substituyen a la religión para legitimar unos valores previamente legitimados por ella.

Ciertamente, en cada una de estas dimensiones del proceso de secularización cabe hablar de innovaciones. Pero se trata de innovaciones que vienen de algún modo impuestas desde fuera: su origen es exógeno, y no endógeno; son innovaciones en la medida en que pueden ser llamadas así unos procesos de adaptación.

Si bien ciertos autores afirman que el proceso de secularización fue desencadenado por la propia tradición religiosa judeo-cristiana, en cuyo caso quedaría demostrada la capacidad de la religión de actuar como variable independiente, no es menos cierto que este mismo proceso impide hoy que la religión actúe como tal: la religión ha de ser considerada hoy, bajo el impacto de la secularización, como variable dependiente. $Y$ en esta misma medida no cabe hablar ya de la religión como fuente de innovación creadora, antes bien de la religión como sujeta a una innovación adaptativa.

A la misma conclusión nos conduce, lógicamente, el análisis del pluralismo. Ya tenga su origen en una situación de diversidad de tradiciones religiosas, o ya se llegue a él por el camino de la secularización -en la medida en que ésta destruye los antiguos monopolios con la introducción de un sistema de legitimación de tipo no religioso al lado de la antigua legitimación religiosa--, el pluralismo comporta dos consecuencias fundamentales para la problemática de la innovación: la aparición de una situación de competencia entre los distintos sistemas de legitimación, y el fenómeno de privatización de la religión.

Los distintos sistemas de legitimación coexistentes dentro de una misma sociedad se hallan inevitablemente en situación de mutua competencia, originándose lo que algunos autores han calificado de «situación de mercado religioso" ${ }^{21}$. Semejante situación de mercado supone básicamente la nece-

21. Cf. P. Berger, «A Market Model for the Analysis of Ecumenicity», Social Research, Vol. 30, n. ${ }^{\circ} 1,1963$, págs. 77-93. 
sidad en que se hallan los suministradores del producto religioso de regular su competencia (de donde la posibilidad de proceder a un análisis sociológico del movimiento ecuménico desde esta perspectiva, considerándolo en tanto que mecanismo regulador de la competencia), así como de tener en cuenta las preferencias de la clientela y de adaptarse a ellas: los clientes se hallan, en efecto, en la situación de consumidores voluntarios del producto religioso, y éste queda consecuentemente sujeto a la moda.

Por otra parte, el mundo de las evidencias colectivas, necesariamente existente en todo tipo de sociedad, se sitúa cada vez más fuera del ámbito de lo religioso. Con ello la religión se convierte progresivamente, dentro de una sociedad diferenciada y como consecuencia del proceso de desmonopolización de lo religioso, en cuestión privada de cada uno, en materia opinable $\mathrm{y}$ asunto de preferencias personales. La institución religiosa, dirán Berger y Luckmann, pasa de institución primaria a institución secundaria, dada esta intervención en la esfera privada más que en la esfera pública y dada su ubicación económica en el sector del consumo ${ }^{22}$.

Así pues, aunque en otros momentos la religión hubiese podido actuar como variable independiente - $y$ sin negar que pueda acaso actuar de nuevo como tal en el futuro- apareciendo por lo tanto como fuerza capaz de promover una innovación creadora, en la situación actual la innovación religiosa se presenta más bien como adaptación a una sociedad dotada de una pluralidad de sistemas de legitimación y que se ha autonomizado, desposeyendo a la religión - de modo previsiblemente duradero- de todo monopolio dentro del sistema cultural.

\section{Para una aplicación de la teoría a la Iglesia como institución}

Dado que la historia de la sociedad occidental se caracteriza, a diferencia probablemente de todas las demás, por la suprema especialización institucional de la religión en forma de Iglesia ${ }^{23}$, una sociología de la religión, aun sin ser una sociología de la Iglesia como institución y aun rechazando todo enfoque exclusivamente eclesiocéntrico, necesariamente ha de referirse a ella y ha de tomar a menudo sus ejemplos e ilustraciones de las formas de religión organizada existentes en la sociedad que constituye el objeto de la investigación. Recogiendo de forma breve y sintética algunos ejemplos de innovación religiosa directamente relacionados con la Iglesia institucional, quisiéramos concluir tratando de abrir una serie de pistas de reflexión y de eventuales investigaciones ulteriores, pero conscientes al

22. «Aspects sociologiques du pluralisme», art. cit.

23. Según T. LuckmanN; cf. la nota 3. 
mismo tiempo de la imposibilidad de explorarlas con detalle en el marco del presente trabajo.

Si hubiera que elegir una palabra clave para designar aquello en torno a lo cual ha girado principalmente la vida del catolicismo en los últimos diez años, fácilmente se podría elegir el término «aggiornamento». El Concilio Vaticano II estuvo profundamente marcado por esta voluntad renovadora, y el período post-conciliar lo ha estado - y en cierto modo lo sigue estando- por la aplicación de las reformas decididas y por las consecuencias acarreadas por tal voluntad de aggiornamento. Consecuencias, por lo demás, en buena parte inesperadas, en la medida en que el concilio mismo respondía a un impulso espontáneo más que a un análisis detallado de los objetivos propuestos y de sus implicaciones. No se pretenda ver aquí, ni en lo que antecede, ni en lo que sigue, un juicio de valor; no se nos quiera hacer decir que es mejor la espontaneidad que el análisis, o que el análisis es preferible a la espontaneidad. Observamos sólo que las consecuencias serán de tipo distinto en uno o en otro caso; cabe preguntarse, por otra parte, si Juan XXIII hubiese convocado un concilio caso de haber sido sociólogo de la religión-cosa que (y, por una vez, juicio de valor) afortunadamente no era- y caso de haber procedido a un análisis sociológico y prospectivo de las implicaciones a largo plazo de semejante decisión; existen motivos para sospechar que no, o para sospechar por lo menos que las presiones para disuadirle de ello hubiesen sido terribles.

Sea como sea, el término «aggiornamento» resulta sorprendentemente interesante y sugestivo dentro del contexto de nuestro tema: de forma perfectamente involuntaria, en efecto, el término parece dar una clara respuesta a la pregunta: ¿innovación-creación, o innovación-adaptación? ¿Acaso «ponerse al día» no es, efectivamente, sinónimo de adaptarse? Podría objetarse que otra expresión que refleja igualmente esta misma realidad aparece dotada de una connotación parcialmente distinta: la expresión, asimismo muy utilizada, de «signos de los tiempos». Sin embargo, si la connotación es distinta probablemente sea, en definitiva, porque es más vaga. ¿Qué implica, en efecto, decir que «la Iglesia ha de leer los signos de los tiempos»? Siendo nuestro problema la innovación, no se trata de saber si los lee o no los lee: se trataría en todo caso de saber si ha de leerlos o si puede «escribirlos». La innovación creadora estribaría en este caso en hacer surgir nuevos signos; leerlos es finalmente otra forma de adaptación. $\mathrm{O}$, para volver de nuevo a nuestra terminología anterior, la expresión supone una autonomía de los «signos de los tiempos»: y si los signos de los tiempos actúan como variable independiente, la Iglesia es variable dependiente. 
Cabría hacer un análisis de todos los cambios introducidos por el último concilio a partir de esta alternativa: innovación-creación o innovación-adaptación. Limitémonos aquí a un solo ejemplo: la redefinición de la Iglesia como Pueblo de Dios, que supone un cambio muy profundo de la concepción de la Iglesia. Ahora bien; decíamos antes que en una sociedad en la que la institución religiosa se convierte en suministrador de un producto que el individuo, en posición de consumidor, podrá aceptar o rechazar a causa de la existencia de una situación pluralista, se hace preciso amoldarse a los deseos de ese consumidor. Desde esa perspectiva (elaborada al tratar de la secularización y el pluralismo) toda la teología del laicado aparece como un reconocimiento implícito de semejante situación, y como legitimación a posteriori de tal estado de cosas ${ }^{24}$. Si aceptamos como válido el modelo propuesto de mercado religioso para explicar muchos de los fenómenos actuales, la culminación de ese proceso de legitimación en la definición de la Iglesia como Pueblo de Dios aparece como esfuerzo de adaptación a los deseos de la clientela. Para seguir empleando una terminología que no quiere en absoluto ser «irreverente», pero que tiene la ventaja de su gran plasticidad, definir a la Iglesia como «Pueblo de Dios» equivale a reconocer que la Iglesia se ha convertido en una «cooperativa de consumidores».

Por comparación con el protestantismo, el proceso de adaptación dentro del catolicismo es un fenómeno muy reciente, e incipiente aún. Y cabe preguntarse incluso hasta qué punto va a proseguir: el innegable instinto sociológico de los medios llamados «conservadores» no puede sino agudizarse ante las perspectivas que se ofrecen a las instituciones protestantes confrontadas con un movimiento como el de la teología «radical» o «secular» llevada hasta sus últimas consecuencias. Si la solución de los teólogos radicales es indudablemente original (partir del supuesto de que Dios no existe; propugnar la rápida desaparición de las organizaciones eclesiásticas), el problema que las suscita es el mismo con el que venimos enfrentándonos: secularización, pluralismo, pérdida de monopolios, competencia entre los sistemas de legitimación, crisis de credibilidad, etc.: en una palabra, el hecho de que las legitimaciones tradicionales proporcionadas por la institución religiosa han dejado de constituir una evidencia colectiva, y de que la Iglesia ha de esforzarse, por lo tanto, en seguir actuando como instancia legitimadora de tal modo que pueda ser aceptada como tal, por ciertos grupos al menos, dentro de la sociedad.

Frente a semejante problemática existen dos opciones fundamentales:

24. Cf. el capítulo 6 de Para una teoría sociológica de la religión, de P. BERGER, Barcelona, Kairós, 1971. 
ponerse a la defensiva o adaptarse. Las dificultades inherentes a la primera de ambas opciones podrían resumirse diciendo que con ello se pretende el regreso a un modelo de sociedad no diferenciada dentro del contexto de una sociedad diferenciada, o el mantenimiento dentro de esta sociedad diferenciada de un subsistema cultural monolítico: se trataría, en este caso, de un pluralismo de ghettos insularizados. Esta postura defensiva fue en buena parte característica del catolicismo post-tridentino, y sigue siéndolo de ciertos grupos habitualmente calificados de sectarios (protestantes o católicos); dentro de un marco no estrictamente religioso, pero que ciertos sociólogos no dudan en considerar como semi-religioso, el mismo tipo de monolitismo dogmático se manifiesta hoy en ciertos grupos «maoístas» occidentales.

Los teólogos radicales son, por su parte, los máximos exponentes de la segunda opción: una opción que, llevada hasta sus últimas consecuencias, conduce a la «auto-liquidación definitiva de la empresa eclesiásticoteológica como tal» ${ }^{25}$. Lo cual permite prever, aunque sólo sea a título de hipótesis, que no se va a llegar hasta esas consecuencias últimas, y que si bien el proceso de adaptación es irreversible, la empresa teológica va a canalizarse en un futuro próximo por senderos un tanto más moderados.

Ello es importante por cuanto demuestra, no sólo que los procesos actuales de innovación religiosa se inscriben dentro de la categoría adaptativa de la innovación, sino también que todo proceso de innovación - aun adaptativa - no se limita a una puesta al día pura y simple, sino que acarrea una serie de consecuencias y repercute a todos los niveles del funcionamiento de la institución religiosa.

\section{ConClusión}

Sin pretender en absoluto haber explorado todas las pistas posibles, consideramos más modestamente haber contribuido a abrir algunas de las más importantes. Todo este trabajo ha intentado ser una invitación a la reflexión sobre el fenómeno religioso desde una perspectiva determinada: la sociología de la religión. Creemos haber suscitado una serie de cuestiones, y haber aportado un cierto instrumental teórico, conceptual y analítico. Por ello no sacamos, no podemos sacar, conclusiones definitivas; no podemos sino sacar hipótesis de trabajo, a partir de las cuales proseguir la reflexión y el análisis.

25. P. Berger, «A Sociological View of the Secularization of Theology», Journal for the Scientific Study of Religion, Vol. 6, n. 1,1967 , pág. 13. 